\title{
Stored blood - an effective immunosuppressive method for transplantation of kidneys from unrelated donors. An 11-year follow-up
}

M.M. Galvão',

Z.F. Peixinho ${ }^{2}$,

N.F. Mendes ${ }^{2}$ and

E. Sabbaga ${ }^{1}$

\author{
${ }^{1}$ Unidade de Transplante Renal, Hospital das Clínicas, \\ Faculdade de Medicina, Universidade de São Paulo, \\ 05403-010 São Paulo, SP, Brasil \\ ${ }^{2}$ Disciplina de Imunologia, Escola Paulista de Medicina, \\ Universidade Federal de São Paulo, 04023-900 São Paulo, SP, Brasil
}

\begin{abstract}
Correspondence
M.M. Galvão

Unidade de Transplante Renal

Hospital das Clínicas, FMUSP

Av. Eneas de Carvalho Aguiar, 255

05403-010 São Paulo, SP

Brasil

Fax: 55 (011) 852-2633
\end{abstract}

Publication supported by FAPESP.

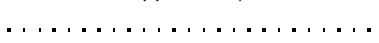

Received August 14, 1996 Accepted April 1, 1997

\section{Abstract}

Thirty-seven patients were submitted to kidney transplantation after transfusion at 2-week intervals with 4-week stored blood from their potential donors. All patients and donors were typed for HLA-A-B and DR antigens. The patients were also tested for cytotoxic antibodies against donor antigens before each transfusion. The percentage of panel reactive antibodies (PRA) was determined against a selected panel of 30 cell donors before and after the transfusions. The patients were immunosuppressed with azathioprine and prednisone. Rejection crises were treated with methylprednisolone. The control group consisted of 23 patients who received grafts from an unrelated donor but who did not receive donor-specific pretransplant blood transfusion. The incidence and reversibility of rejection episodes, allograft loss caused by rejection, and patient and graft survival rates were determined for both groups. Non-parametric methods (chi-square and Fisher tests) were used for statistical analysis, with the level of significance set at $\mathrm{P}<0.05$. The incidence and reversibility of rejection crises during the first 60 post-transplant days did not differ significantly between groups. The actuarial graft and patient survival rates at five years were $56 \%$ and $77 \%$, respectively, for the treated group and $39.8 \%$ and $57.5 \%$ for the control group. Graft loss due to rejection was significantly higher in the untreated group $(\mathrm{P}=0.0026)$ which also required more intense immunosuppression $(\mathrm{P}=0.0001)$. We conclude that tranfusions using stored blood have the immunosuppressive effect of fresh blood transfusions without the risk of provoking a widespread formation of antibodies. In addition, this method permits a reduction of the immunosuppressive drugs during the process without impairing the adequate functioning of the renal graft.
Key words

- Kidney transplantation

- DST

- Stored blood 


\section{Introduction}

In the early eighties, a time when blood tranfusion protocols were still used as a means of patient preparation for kidney transplantation (1), we conducted an investigation involving the possible effects of pretransplant blood transfusions using whole donor-specific stored blood as a means of reducing the degree of patient allogenic sensitization observed after tranfusion of fresh blood. Blood storage was first reported by Opelz et al. in 1981 (2). The effect of reduced sensitization seemed to be related to the loss of leucocytes and platelets containing large amounts of HLA antigens.

Light et al. $(3,4)$ analyzed the characteristics of leucocytes obtained from serial units of human blood stored for a minimum period of 3 days which were found in a progressively reduced number as storage time increased. The serial study of cells obtained by separation using a Ficoll Hypaque gradient and stained with acid peroxidase revealed that up to the 8th day of storage most cells were found to be degranulated neutrophils and not mononuclear cells. Analysis of such cellular preparation using a "fluorescence activated cell sorter" (FACS) indicated the rapid loss of $\mathrm{T}$ lymphocytes, granulocytes and platelets with preservation of B lymphocytes and monocytes. After 2-week storage less than $15 \% \mathrm{~T}$ lymphocytes (OKT3) (cells containing large amounts of class I MHC antigens) were present in the samples. The study of immunogenicity revealed that cells containing large amounts of class II MHC antigens (monocytes and B lymphocytes) had a stimulating activity in a mixed lymphocyte culture (MLC) and produced a strong response in a sensitized lymphocyte assay (PLT) throughout the storage time (30 days).

In parallel, an in vivo experiment carried out with Rhesus monkeys indicated that practically all animals submitted to three whole blood transfusions using stored blood (up to 2 weeks) developed lymphocytotoxic anti- bodies compared to about $50 \%$ of the monkeys transfused with blood stored for 3 or 4 weeks. Although the degree of RhLA incompatibility between donor and recipient of blood transfusions has not been mentioned, this finding indicated an inverse relationship between blood immunogenicity and storage time (5). Nevertheless, the question rose of whether stored blood would be as effective as fresh blood to enhance allograft survival. Later, the same investigators (6) demonstrated that previous blood transfusions using stored blood inhibit the sensitization of Rhesus monkeys to fresh blood, suggesting a possible immunomodulating effect on the immune response.

In our experience, this program involving three donor-specific blood transfusions using blood stored for four weeks and transfused into unrelated recipients proved to be successful since the observed sensitization rate was only $4.1 \%$ when evaluated by the standard NIH crossmatch technique (7). Patients thus treated received the kidney from their specific donors.

The objective of the present study was to analyze the evolution of these patients by comparing them with a control group of patients who had received grafts from unrelated donors without previous donor-specific blood transfusions.

\section{Patients and Methods}

\section{DST-treated group}

Starting in 1983, 37 patients were submitted to kidney transplantation and received grafts from unrelated living donors after three blood transfusions using blood stored for four weeks, a procedure known as donorspecific blood transfusion (DST).

All patients and donors underwent HLAA-B and DR antigen typing and the recipients were tested for anti-donor antigen cytotoxic antibodies prior to each transfusion. The percentage of panel reactive antibodies 
(PRA) was determined before and at the end of the blood transfusions. Transplantation was performed about 19 days after the last blood transfusion.

\section{Transfusions}

Two hundred ml blood was obtained from the donor in CPDA-1 (citrate-phosphate-dextrose-adenine) and stored at $4^{\circ} \mathrm{C}$ for four weeks prior to transfusion. The transfusions were performed at 15-day intervals. The patients were submitted to the usual hemodialysis session at least $48 \mathrm{~h}$ after each blood transfusion. Occasional dialysis transfusions were interrupted 15 days prior to the beginning of the program.

\section{Immunosuppressive therapy}

As post-transplant immunosuppressive therapy the patients received only azathioprine and prednisone. In some cases of low tolerance (hepatotoxicity or myelotoxicity), azathioprine was replaced with cyclosporine A. Rejection episodes were treated with methylprednisolone pulses (1 g/day for 3 days).

Azathioprine was instituted $48 \mathrm{~h}$ before kidney transplantation at a dose of $5 \mathrm{mg} \mathrm{kg}^{-1}$ day $^{-1}$. On the third postoperative day the dose was reduced to $3 \mathrm{mg} \mathrm{kg}^{-1}$ day $^{-1}$. The maintenance dose after one year ranged from 0.22 to $2.8 \mathrm{mg} \mathrm{kg}^{-1}$ day $^{-1}$ depending on factors such as tolerance, age, early post-transplant evolution, and data concerning pretransplant immunologic condition (retransplant, number of random transfusions, panel and number of previous pregnancies). Six patients were maintained on a dose of $1 \mathrm{mg}$ $\mathrm{kg}^{-1}$ day $^{-1}$ azathioprine or less for a period of 3 to 60 months.

Prednisone therapy was initiated on the day of transplantation at a dose of $1.5 \mathrm{mg} \mathrm{kg}^{-1}$ day $^{-1}$ and was slowly reduced to a maintenance dose of 12.5 to $15 \mathrm{mg} /$ day one year after transplantation, and 7.5 to $12.5 \mathrm{mg} /$ day two years postoperatively. Three patients were maintained on corticosteroid monotherapy for 3, 6 and 30 months. Patients on monotherapy or on low doses of azathioprine ( $<1 \mathrm{mg} \mathrm{kg}^{-1} \mathrm{day}^{-1}$ ) received higher doses of prednisone (15 to $20 \mathrm{mg} /$ day).

In cases requiring cyclosporine the doses were established for each patient so as to obtain serum concentrations of $120 \mathrm{ng} / \mathrm{ml}$ or lower.

\section{Control group}

The control group consisted of 23 patients who received grafts from unrelated living donors but who did not undergo donor-specific pretransplant blood transfusion. A double immunosuppressive scheme (azathioprine and prednisone) was used in 5 patients, and a triple therapy (azathioprine, prednisone and cyclosporine) was used in 18 patients. Two of these 18 patients also received monoclonal antibodies. Time since transplantation coincided with that of patients receiving stored blood. The two groups were compared regarding age, sex, dialysis time, total number of random transfusions and previous pregnancies, previous transplantation and immunosuppressive therapy (Table 1). The incidence and reversibility of rejection episodes, allograft loss caused by

Table 1 - Comparison between DST-treated (transfused group) and control (nontransfused group) patients.

*Two of 18 patients received quadruple therapy. NS, Nonsignificant.

\begin{tabular}{|c|c|c|c|}
\hline Parameters & DST-treated $(\mathrm{N}=37)$ & Control $(N=23)$ & $P$ value \\
\hline Age (years) & $39.41 \pm 10.77$ & $42.00 \pm 13.63$ & NS \\
\hline Male/female & $21 / 16$ & $18 / 5$ & NS \\
\hline Time on dialysis (months) & $28.63 \pm 13.22$ & $19.40 \pm 15.59$ & NS \\
\hline $\begin{array}{l}\text { Random transfusions/patient } \\
\text { (number of transfused patients) }\end{array}$ & $28 / 37$ & $20 / 23$ & NS \\
\hline Pregnancies & $32 / 12$ & $0 / 5$ & 0.0062 \\
\hline Retransplant & $2 / 37$ & $5 / 23$ & NS \\
\hline $\begin{array}{l}\text { Immunosuppressive therapy } \\
\text { double }\end{array}$ & $34 / 37(91.8 \%)$ & $5 / 23(21.7 \%)$ & 0.0001 \\
\hline triple & $3 / 37(8.1 \%)$ & $18 * / 23(78.2 \%)$ & 0.015 \\
\hline
\end{tabular}


Table 2 - Evolution of DST-treated (transfused group) and control (nontransfused group) patients.

NS, Nonsigificant.

\begin{tabular}{lccc}
\hline Events & $\begin{array}{c}\text { DST-treated } \\
(\mathrm{N}=37)\end{array}$ & $\begin{array}{c}\text { Control } \\
(\mathrm{N}=23)\end{array}$ & P value \\
\hline Early rejections & $25 / 17$ & $26 / 15$ & $\mathrm{NS}$ \\
(crisis/patient) & $(45.9 \%)$ & $(65.2 \%)$ & \\
Reversed & $24 / 25$ & $22 / 26$ & $\mathrm{NS}$ \\
crises & $(96.0 \%)$ & $(84.6 \%)$ & \\
Late & $6 / 37$ & $10 / 23$ & 0.0432 \\
rejections & $(16.2 \%)$ & $(43.4 \%)$ & \\
Kidney loss & $3 / 37$ & $10 / 23$ & 0.0026 \\
by rejection & $(8.1 \%)$ & $(43.4 \%)$ &
\end{tabular}

rejection, and patient and graft survival rates were determined for both groups.

Non-parametric methods (chi-square and Fisher tests) were used for statistical analysis, with the level of significance set at $\mathrm{P}<0.05$.

\section{Results}

There was no significant change in percent reactivity to the panel between the results obtained before and after transfusion. In two patients the crossmatch became negative following blood transfusion.

\section{Rejection episodes}

The incidence and reversibility of rejection crises during the first 60 post-transplant days (early rejections) did not differ significantly between groups. Two months posttransplant (late rejection), six rejection crises occurred in the treated group (6/37; $16.2 \%$ ), four of which were due to interruption of immunosuppressive therapy, and one to cytomegalovirus infection. There were three complete reversals. Ten rejection episodes with three partial reversals were observed in the control group $(10 / 23 ; 43.4 \%)$ $(\mathrm{P}=0.0432)$ (Table 2). Rejection episodes after two months were significantly less frequent in the DST-treated group.

\section{Survival rates}

The actuarial graft and patient survival rates at five years were $56 \%$ and $77 \%$, respectively, for the treated group and $39.8 \%$ and $57.5 \%$ for the control group (Figure 1B and $1 \mathrm{~A})$.

Graft loss due to rejection was significantly higher in the control group. In the DST-treated group there were eight deaths during the study period, all involving patients with functioning grafts, and seven graft losses, only three $(3 / 37 ; 8.1 \%)$ of which were due to rejection consequent to discontinuation of immunosuppressive therapy. In the control group, twelve patients died, four with their renal function preserved. The remaining control patients showed chronic rejection and three allograft losses, one of which was due to nephrotoxicity resulting from cyclosporine treatment $(10 / 23 ; 43.4 \%)$ (Table 2). There was a considerably higher number of graft losses due to rejection in the control group.

\section{Retransplant}

In the DST-treated group, two patients were retransplanted and both maintained normal renal function 87 and 99 months after transplantation. In the control group, five patients were retransplanted. Of these, one lost the kidney due to acute transplant vasculopathy 17 days after transplantation, and two lost the graft due to chronic rejection 18 and 48 months after transplantation. In the DST-treated group the patients submitted to retransplant had a better outcome than the retransplanted patients in the control group.

\section{Monotherapy and low doses of immunosuppressive drugs}

The three patients in the transfused group maintained on monotherapy with prednisone (Figure 2A,B,C) or those who were kept on 
low doses of azathioprine $\left(<1 \mathrm{mg} \mathrm{kg}^{-1} \mathrm{day}^{-1}\right)$ did not show changes in renal function during this period of treatment. It should be noted that the DST-treated patients needed fewer immunosuppressive drugs (double therapy, 34/37;91.8\%) than the control group $(5 / 23 ; 21.7 \%)$, in addition to tolerating lower doses during treatment.

\section{Discussion}

The protocols used by Light et al. (8) and Whelchel et al. (9) for humans, although based on transfusions that were specific for the donor of the kidney transplant, did not permit an evaluation of the effect of time of blood storage on the survival of the renal allograft. The first group of authors administered to each patient three whole blood transfusions using blood stored for 1, 3 and 5 weeks; the second group of investigators performed blood transfusions using blood with $2,14,21$ and 28 days of storage. In both cases, the initial tranfusion was found to have caused enough allogenic stimulation (immunogenic) to prevent a possible tolerogenic effect caused by later transfusions.

Our patients received three donor-specific transfusions of blood stored for identical periods of time. The low incidence of rejection episodes during the first 60 post-transplant days and the good response to standard therapy suggest a protective effect of stored blood transfusion during the early post-transplant period. It is known that after transfusion there occurs an increase in the production of PGE-2 by macrophages, with this increase being more pronounced the longer the storage of the transfused product $(10,11)$. Prostaglandin levels usually remain high for three months post-transfusion (12). An analogous fact occurs regarding function of the suppressor cells whose activity intensifies after blood transfusion, returning to former levels four weeks after transfusion $(10,13,14)$.

These findings led us to believe that transplantation should be performed soon after the last transfusion for the patient to benefit from the nonspecific protection of the mediators induced by transfusion. Furthermore, since we are dealing here with potentially sensitized patients, adequate immunosuppression should be provided during the early post-transplant period to assure the deletion of the immunologically active cellular clones, thus permitting the anti-idiotype antibodies to develop $(15,16)$.

The number of late rejections was significantly smaller in the transfused group than in the nontransfused group $(\mathrm{P}=0.0432)$ and the same was observed regarding graft losses due to rejection (treated group: $8.1 \% \mathrm{vs}$ $43.4 \%$ in control group, $\mathrm{P}=0.0026$ ).

The effect of transfusion seems to be long lasting. In the present study it was pos-
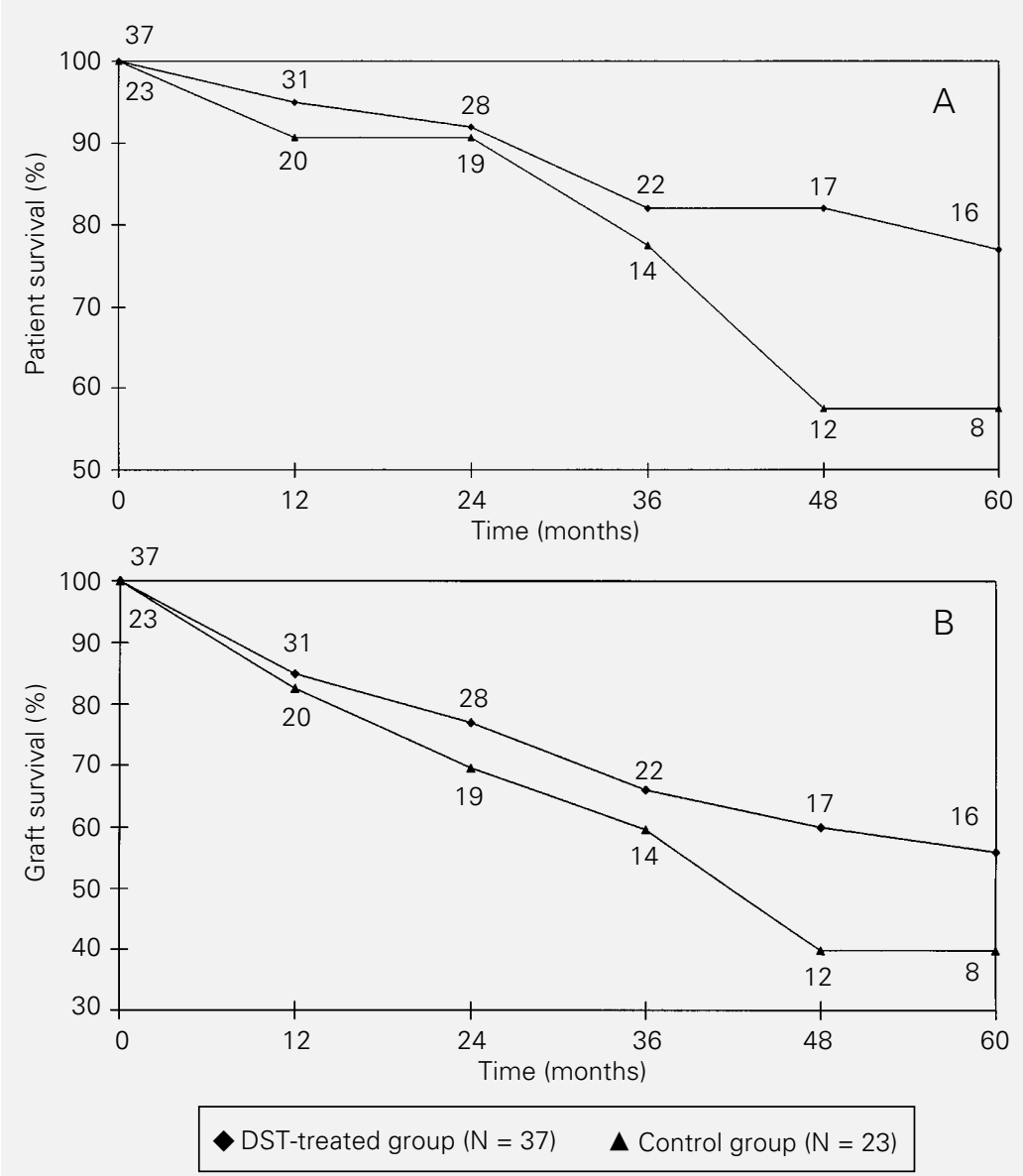

Figure 1 - $A$, Actuarial patient survival. $B$, Actuarial graft survival. 
sible to keep some of the patients on reduced doses of azathioprine, and three on monotherapy with corticosteroids for a period of 3 to 30 months.

Other investigators have suggested $(17,18)$ that a certain HLA mismatching grade (non-HLA-sharing) between donor and recipient, mainly concerning class II antigens, is necessary to obtain the transfusion effect
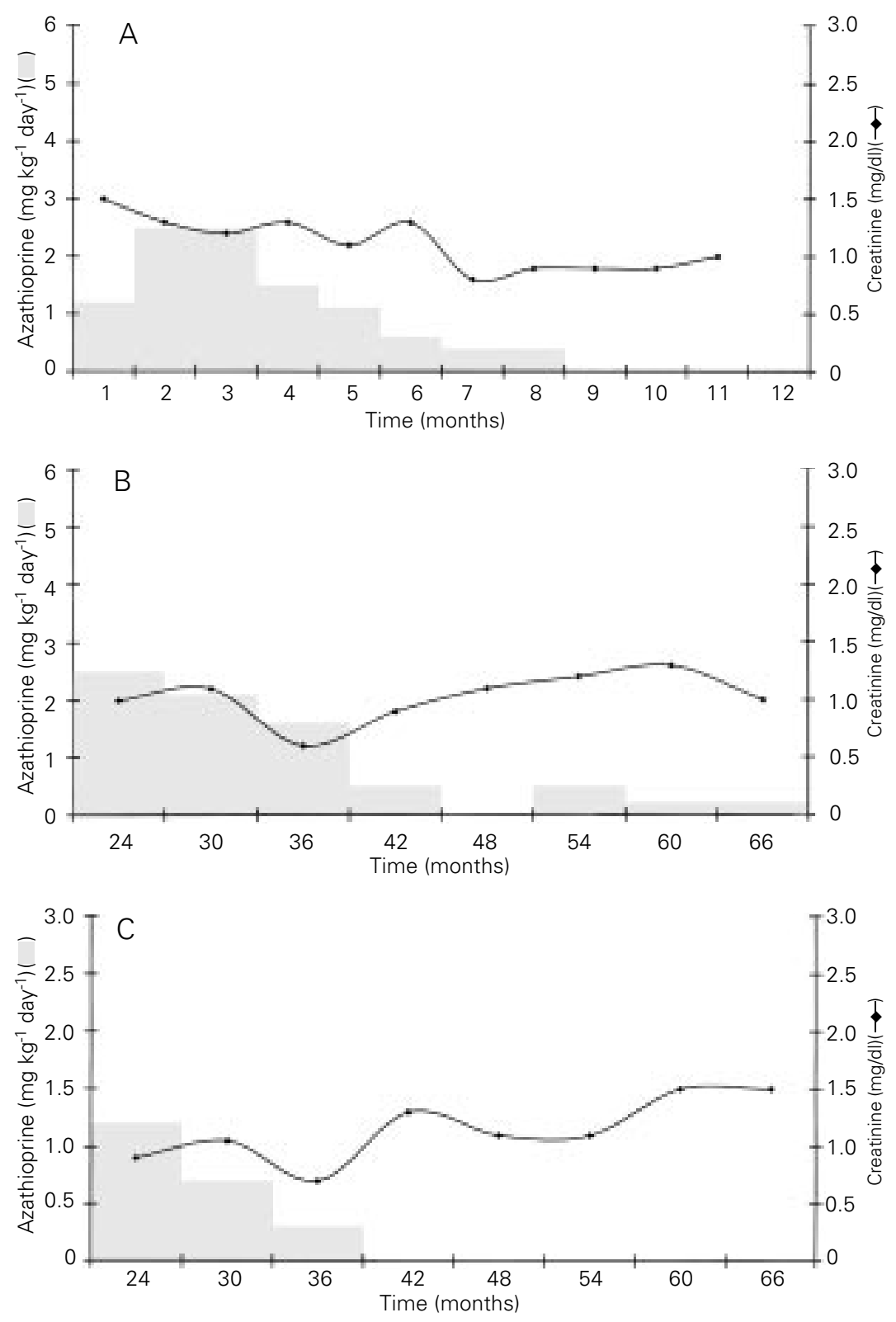

Figure 2 - Transfused patients maintained on monotherapy with prednisone for 3 (A), 6 (B) and 30 (C) months. and to induce enhancement of allograft survival. The protocol reported here is probably more suitable for transplantation involving non-consanguineous individuals.

Blood is a multisubstance complex, and consequently is apt to interfere with the immune response in several ways. Transfusion of erythrocytes can result in immunologic unresponsiveness and a better outcome for the transplant (19). The senescent red blood cell exposes an antigen (neoantigen) that makes it immunogenic. Immunoglobulins, especially $\mathrm{IgG}$, attach themselves to the cell membrane rendering the cell vulnerable to phagocytosis. The neoantigen that emerges in the senescent erythrocyte also occurs on other somatic cells including cells of the proximal renal tubule $(20,21)$.

The quantity of IgG found on the erythrocyte surface increases with storage time. The neoantigen is likely to play a role in the effect of tranfusion using stored blood. Antigen features and means of display most probably are important for its effect on the immune response. When we consider the fact that, upon administration of stored blood, ruptured leukocytes and red blood cells are being transferred into the recipient it is reasonable to assume that a large amount of class I soluble antigens, once in the blood stream, are able to promote the appropriate emergence of antigens so as to induce tolerance.

Stored blood contains microaggregates of fibrin residues, degenerate leukocytes and blood platelets that bind to fibronectin causing a significant decrease in serum concentrations of this molecule, and consequently leading to insufficient macrophage activity. Besides playing a role in macrophage function, fibronectin is a surface molecule closely involved in cell adhesion, activation and migration $(22,23)$.

Mincheff et al. (24) have shown that blood stored for 13 or more days loses its immunogenicity because its ability to present the accessory signal diminishes, with a 
consequent induction of some degree of immunosuppression.

Blood storage and the resulting loss of ability by presenting cells (APC) to express the costimulatory signals impair the direct presentation of alloantigens to recipient $\mathrm{T}$ cells. The indirect presentation by recipient APC is important whether alloantigens are in a solubilized form or expressed on the membrane of donor cells. As the storage of blood progresses the cells undergo secondary necrosis with a loss of membrane integrity. Mincheff et al. (25), working with an inbred rodent model, transfused intravenously necrotic, apoptotic or viable allogeneic cells and achieved immunosuppression after administration of necrotic cells.
Probably several factors intervene in the effect of transfusion with variable intensity and at different moments after the transplant. We conclude that the present method allows a reduction of immunosuppressive drugs without risking the good function of the renal allograft. The practical implication of good results with DST using stored blood is the availability of a larger number of potential living donors for kidney transplantation.

\section{Acknowledgment}

We are grateful to the Fundação PróSangue, Hemocentro, São Paulo, for blood storage and transfusions.

\section{References}

1. Salvatierra $O$, Iwaki $Y$, Vincent $P$, Amend W, Potter D, Opelz G, Terasaki P, Duca R, Hopper S \& Feduska N (1981). Incidence, characteristics and outcome of recipients sensitized after donor-specific blood transfusions. Transplantation, 32: 528531.

2. Opelz G, Graver B, Mickey MR \& Terasaki PI (1981). Lymphocytotoxic antibody responses to transfusions in potential kidney transplant recipients. Transplantation, 32: 177-183.

3. Light JA, Metz S, Oddenino K, Simonis T, Strong DM, Reinmuth B, Kümar J \& Biggers JA (1982). Fresh versus stored blood in donor specific transfusions. Transplantation Proceedings, 14: 296-301.

4. Light JA, Metz S, Oddenino K, Strong DM, Simonis T, Biggers JA \& FernandezBueno C (1982). Donor specific transfusion with diminished sensitization. Transplantation, 34: 352-355.

5. Oh JH, McClure HM \& Galvão MM (1982). Comparison of immunogenicity of fresh and stored blood - A study in Rhesus monkeys. Transplantation, 33: 198-200.

6. Galvão MM, Tuttle EP, Oh JH \& McClure HM (1984). Lymphocytotoxic donor-specific response to fresh and stored blood An experiment in immunosuppressed Rhesus monkeys. International Urology and Nephrology, 16: 253-257.
7. Galvão MM, Lima MG, Persole LB, Mendes NF \& Sabbaga E (1987). Diminuição do índice de sensibilização com transfusão de sangue armazenado doador-específico. Revista do Hospital das Clínicas da Faculdade de Medicina de São Paulo, 42: 305-307.

8. Light JA, Metz SJ \& Oddenino K (1983). Donor-specific transfusion with minimal sensitization. Transplantation Proceedings, 15: 917-923.

9. Whelchel JD, Shaw JF, Curtis JJ, Luke RG \& Diethelm AG (1982). Effect of pretransplant stored donor-specific blood transfusions on early renal allograft survival in one haplotype living related transplants. Transplantation, 34: 326-329.

10. Francis DMA (1991). Relationship between blood transfusion and tumour behaviour. British Journal of Surgery, 72: 1420-1428.

11. Alexander JW (1991). Transfusion-induced immunomodulation and infection. Transfusion, 31: 195-196.

12. Lenhard V, Gemsa D \& Opelz G (1985). Transfusion-induced release of prostaglandin $E_{2}$ and its role in the activation of T-suppressor cells. Transplantation Proceedings, 6: 2380-2382

13. Smith MD, Williams JD, Coles GA \& Saleman JR (1981). The effect of blood transfusion on T-suppressor cells in renal dialysis patients. Transplantation Proceedings, 1: 181-183.
14. Gafter V, Kalechman Y \& Sredin B (1992). Induction of a subpopulation of suppressor cells by a single blood transfusion. Kidney International, 41: 143-148.

15. Waal LP, Twuyver E, Mooijaart RJD, Lardy NM, Kast WM \& Melief CJM (1991). Mechanisms of transplantation tolerance: Do anti-idiotypic antibodies play a role? Transplantation Proceedings, 23: 155-156.

16. Downey WE, Baldwin WM \& Sanfilippo F (1990). Association of donor-specific blood transfusion enhancement of rat renal allografts with accelerated development of antiidiotypic antibodies and reduced alloantibody responses. Transplantation, 49: 160-166.

17. Lazda VA, Pollak R, Mozes MF, Barber PL \& Jonasson O (1990). Evidence that HLA class II disparity is required for the induction of renal allograft enhancement by donor-specific blood transfusions in man. Transplantation, 49: 1084-1087.

18. Bektas H, Klempnauer J \& Jörns A (1993) Differences in immunologic unresponsiveness after MHC-incompatible heart transplantation induced by donor-specific blood transfusion. Transplantation Proceedings, 25: 2870-2871.

19. Keown PA \& Descamps B (1979). Improved renal allograft survival after blood transfusion: a nonspecific erythrocyte-mediated immunoregulatory process? Lancet, 1: 20-22. 
20. Kay MMB (1986). Senescent cell-antigen - A red cell aging antigen. In: Garraty G (Editor), Red Cell Antigens and Antibodies. American Association of Blood Banks, Arlington, VA, 35-80.

21. Kay MMB (1974). Mechanism of removal of senescent red cells. Gerontologist, 14: 33-48.
22. Snyder EL, Mosher DF, Hezzey A \& Golenusky G (1981). Effect of blood transfusion on in vivo levels of plasma fibronectin. Journal of Laboratory and Clinical Medicine, 98: 336-341.

23. Saba TM, Blumenstock FA \& Landaburu $\mathrm{RH}$ (1986). Reversal of opsonic deficiency in surgical, trauma and burn patients by infusion of purified human plasma fibronectin. American Journal of Medicine, 80 : 229-240.
24. Mincheff MS, Meryman HT, Kapoor V, Alsop P \& Wötzel M (1993). Blood transfusion and immunomodulation: A possible mechanism. Vox Sanguinis, 65: 18-24.

25. Mincheff MS, Getsov SI \& Meryman HT (1995). Mechanisms of alloimmunization and immunosuppression by blood transfusions in an inbred rodent model. Transplantation, 60: 815-821. 